\title{
MSE and Tracking of Channel Estimation Based on DD-EW-RLS for Indoor and Outdoor Environment in MIMO-OFDM
}

\author{
Suzi Seroja Sarnin and Siti Maisurah Sulong
}

\begin{abstract}
This paper is focusing on the performance of the proposed EW-RLS channel estimator using simulations, in the context of communication system with MIMO antenna operating in outdoor and indoor environments. Different training rates and different Doppler frequencies are used to track time-variations of the channel. The adaptive algorithm is namely Recursive Least Square (RLS) algorithm. The performance is evaluated in system MSE, for different Doppler frequencies (correspond to different mobility speeds). Simulation results have demonstrated that time-domain adaptive channel estimation and tracking in MIMO OFDM systems based on the DD-RLS is very effective in slowly to moderate time-varying fading channels. This paper provides analysis, evaluation and computer simulations in MATLAB
\end{abstract}

Index Terms-Means square error, multiple input multiple output, orthogonal frequency division multiplexing, recursive least square.

\section{INTRODUCTION}

MIMO refers to the use of an array of antennas for both transmitting and receiving. It has great capability to improve wireless system performance and overcome multipath fading which commonly occur in communication link. Diversity of the recceived signal will increase when the number of antenna increase. Transmission is divided over the antennas to increase array gain, thus improves spectral efficiency and link reliability resulting in diversity gain in the system.

Later, a combination of MIMO with OFDM was proposed due to the channel exhibits frequency selectivity that can cause interference in the system. OFDM is similar to conventional frequency division multiplexing (FDM) and recently been applied widely in wireless communication system due to its robustness to multipath delay [1]. This type of modulation technique uses several orthogonal sub-carriers to transmit and receive high transmission rate signal [2]. Orthogonality in communication means uncorrelated signals with no interference between carriers. The primary advantage of OFDM over single-carrier schemes is its ability to cope with severe channel conditions without complex equalization filters. OFDM communications systems are able to more effectively utilize the frequency spectrum through overlapping sub-carriers.

Manuscript received December 22, 2014; revised March 6, 2015. This work was supported in part by the Faculty of Electrical Engineering UiTM Shah Alam Selangor Malaysia.

The authors are with Faculty of Electrical Engineering UiTM Shah Alam, Malaysia (e-mail:

sitimaisurah91@gmail.com)
Even though channel estimation is challenging problem in any communication system especially when the channel is time-varying, but it still helpful to improve accuracy of the received signal. Many channel estimation techniques have been proposed for MIMO OFDM systems. In [3], the basic Least Mean Square (LMS) and RLS algorithm for 4G MIMO OFDM system had been proposed to evaluate their performance and proved that RLS perform better than LMS but suffer for its computational complexity. To provide robustness in environment and exhibits a fast convergence rate, variable step-size sign algorithm had been proposed in [4]. Instead of using RLS, they were using LMS due to its simplicity. For [5], the researchers also use LMS algorithm and implement their LMS channel estimator for WiMAX physical layer. The reason for using LMS is because of its low complexity. This paper will proposed decision directed exponentially weight RLS for indoor and outdoor environment of channel estimation for MIMO OFDM. The system will be evaluated in terms of MSE and tracking performance.

\section{Methodology}

Channel estimation will perform in time domain first before changing to frequency domain using zero forcing equalization technique. A first order Markov process will be used to describe time-variations of the channel tap. Mobile receiver is moving at speed $60 \mathrm{Km} / \mathrm{h}$ with maximum length $L=4$ taps in Rayleigh fading channel. For indoor scenario, the mobile speed is roughly $18 \mathrm{Km} / \mathrm{h}$ while outdoor is $34 \mathrm{Km} / \mathrm{h}$ by assuming radio frequency is $2.4 \mathrm{GHz}$.

\section{A. System Parameter}

In order to minimize error propagation, training symbols are inserted in time-domain for the duration of one OFDM symbol. It will be periodically transmitted from each antenna. Channel parameters are estimated by using DD-EW-RLS algorithm after receiving the training symbols. After switching the estimator to work in the DD-mode, the variations occur during data transmission can be tracked.

Assume a 2-transmit and 2-receive MIMO OFDM system applied for this research. Table I below shows system parameter used for this research simulation.

\section{B. Outdoor Channel Estimation and Tracking Based on DD-EW-RLS Scheme}

Table II shows values of forgetting factor used for outdoor simulation. Based on the Table III, four numbers of paths had been chosen with different delay and power. The path powers 
are calculated using equation in [6].

TABLE I: MIMO OFDM SYSTEM PARAMETER SIMULATION [6]
\begin{tabular}{|l|l|}
\hline \multicolumn{1}{|c|}{ Parameter } & \multicolumn{1}{|c|}{ Value } \\
\hline Channel bandwidth (BW) & $1 \mathrm{MHz}$ \\
\hline Sampling frequency (fs) & $1 \mathrm{MHz}$ \\
\hline RF frequency (fc) & $2.4 \mathrm{GHz}$ \\
\hline Channel Length $(\mathrm{L})$ & $4 \mathrm{taps}$ \\
\hline FFT size (NFFT) & 128 \\
\hline Number of subcarriers $(\mathrm{K})$ & 128 \\
\hline Subcarrier spacing $(\Delta \mathrm{f})$ & $7.813 \mathrm{kHz}$ \\
\hline Cyclic prefix length $(\mathrm{Ncp})$ & $8 \mathrm{samples}$ \\
\hline Modulation type & QPSK \\
\hline Useful symbol duration $(\mathrm{TB})$ & $128 \mu \mathrm{s}$ \\
\hline OFDM symbol duration $(\mathrm{Ts})$ & $136 \mu \mathrm{s}$ \\
\hline Maximum delay spread $(\tau \mathrm{max})$ & $3 \mu \mathrm{s}$ \\
\hline
\end{tabular}

TABLE II: OPTIMUM VALUES OF THE FORGETTING FACTOR FOR OUTDOOR

\begin{tabular}{|c|c|c|c|}
\hline Training rate & $\mathbf{1 0 \%}$ & $\mathbf{4 \%}$ & $\mathbf{2 \%}$ \\
\hline $\mathrm{f}_{\mathrm{d}}=40 \mathrm{~Hz}$ & 0.992 & 0.995 & 0.997 \\
\hline $\mathrm{f}_{\mathrm{d}}=75 \mathrm{~Hz}$ & 0.991 & 0.993 & 0.995 \\
\hline
\end{tabular}

TABLE III: Multipath Channel Profile

\begin{tabular}{|c|c|c|}
\hline $\begin{array}{c}\text { Path index, } \\
\qquad\end{array}$ & $\begin{array}{c}\text { Path } \\
\text { delay, } \\
\tau_{1}(\mu s)\end{array}$ & $\begin{array}{c}\text { Path power, } P_{1} \\
\text { (dB) }\end{array}$ \\
\hline 1 & 0 & 0.0 \\
\hline 2 & 1.0 & -2.15 \\
\hline 3 & 2.0 & -3.058 \\
\hline 4 & 3.0 & -4.045 \\
\hline
\end{tabular}

\section{Indoor Channel Estimation and Tracking Based on DD-EW-RLS Scheme}

TABLE IV: OPTIMUM VALUES OF THE FORGETTING FACTOR FOR INDOOR

\begin{tabular}{|l|l|l|l|}
\hline Training rate & $\mathbf{2 5 \%}$ & $\mathbf{1 0 \%}$ & $\mathbf{4 \%}$ \\
\hline $\mathrm{F}_{\mathrm{d}}=10 \mathrm{~Hz}$ & 0.995 & 0.995 & 0.997 \\
\hline $\mathrm{F}_{\mathrm{d}}=50 \mathrm{~Hz}$ & 0.995 & 0.995 & 0.997 \\
\hline $\mathrm{F}_{\mathrm{d}}=100 \mathrm{~Hz}$ & 0.995 & 0.995 & 0.997 \\
\hline $\mathrm{F}_{\mathrm{d}}=200 \mathrm{~Hz}$ & 0.995 & 0.995 & 0.997 \\
\hline
\end{tabular}

The optimum forgetting factor $\lambda$ is selected via simulations for different training rates, as is given in Table IV.

\section{MIMO Channel Tracking Based on the First-Order Markov Model}

In wireless mobile communication systems the channel is time-varying which requires the adaptive algorithm of the channel estimation to possess an acceptable tracking capability. To effectively track the time-variations of the channel parameters using the EW-RLS algorithm, the forgetting factor $\lambda$ should be in the range $0.9 \leq \lambda \leq 1$. Another method to track the channel parameter variations is to use a statistical model that describes the dynamics of the channel impulse response taps. Assuming that the time variation of the channel taps is slow enough and that all the taps fade at the same Doppler rate fDmax, it may be most probably to describe such parameter variations by first-order Markov process as

$$
h(n+1)=A h(n)+\omega(n)
$$

where $h(n)$ is the optimum channel estimate at time $n$ while $\omega(n)$ is the process noise vector. $A=a I$ is known state transition matrix with $a=J\left(2 \pi f_{D \max } T_{s}\right)$ is a constant parameter. $T_{s}$ is the OFDM symbol duration which also indicate the constant parameter is very close to unity.

\section{E. RLS Channel Estimator}

Time domain RLS channel estimator can be derived after defining the estimation error and cost function. The estimation error can be defined as

$$
e_{q}(i)=y_{q}(i)-y_{q}(i)=y_{q}(i)-\tilde{X}(i) h_{n, q}
$$

when $h_{n}$ time varying tap-weight vector, $y_{q}(i)$ is the difference between the noisy received signal $y_{q}(i)$ is the estimated output. $\tilde{X}(i)$ is a $1 \times \mathrm{Mt} \mathrm{L}$ vector representing the inputs, from the reference training sequences, into the adaptive filter at the relative instant $i$, and is given by

$$
\begin{gathered}
\tilde{X}(i)=\left[x_{1}(i), x_{2}(i), \ldots, x_{M t}(i)\right. \\
x_{p}(i)=\left[x_{p}(i), x_{p}(i-1), \ldots, x_{p}(i-L+1)\right]
\end{gathered}
$$

In a matrix -vector form, $e_{q}(i)$ can be defined as

$$
e_{q}(n)=\tilde{y}_{q}(n)-\tilde{X}(n) \tilde{h}_{n, q}
$$

where $e_{q}(n)$ is an $[(n+1) \times 1]$ vector of the instantaneous errors, $\tilde{y}_{q}(n)$ is an $[(n+1) \times 1]$ vector of the noisy received signal, $\tilde{X}(n)$ is an $\left[(n+1) \times M_{t} L\right]$ input matrix and $\tilde{h}_{n, q}$ is a $\left(M_{t} L \times 1\right)$ vector that contains the stacked vectors of the channel impulse responses from the transmit antennas to the $q$ th receive antenna, which are under estimate at time instant $n$, with $\mathbf{h}_{n, p q}$ denotes a $L \times 1$ vector of the channel impulse response between the $p$ th transmit and $q$ th receive antennas.

\section{SimULATION RESUlTS}

\section{A. Indoor Channel Estimation and Tracking}

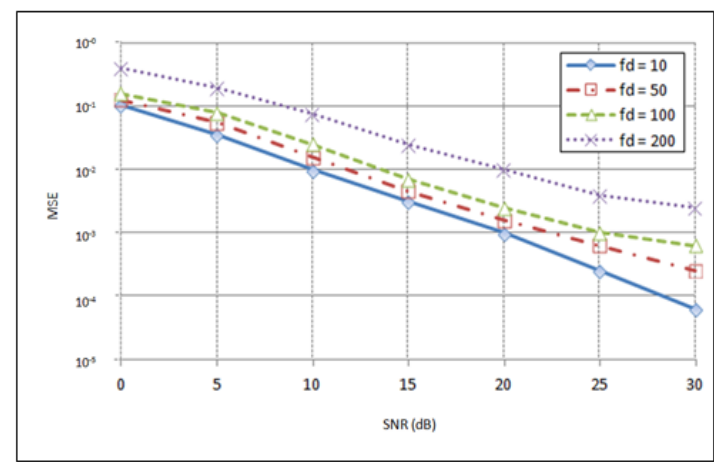

Fig. 1. MSE of channel estimates of the DD EW-RLS estimator with $25 \%$ training data.

Fig. 1 - Fig. 3 show the MSE of the channel tracking versus SNR for different Doppler frequencies and for training rates 
of $25 \%, 10 \%$, and $4 \%$, respectively. It can be noticed that the MSE performance of the estimator is almost the same when the channel is experiencing low Doppler frequencies, regardless of training rate. However, very small degradation in the MSE of the channel estimates is noticed as the Doppler frequencies increases and the training rates decreases, especially at higher SNR. These increases in the MSE pertain to the operation of the estimator in the DD-mode and to the increase in the mobile velocity. However, since the time variations of channel parameters are slow, the movement of the receiver creates only a minor contribution to the total MSE and major effect is due the accumulated decision errors created during the operation of channel tracker in the DD-mode. The estimator tracking capabilities may be quite improved if error correlation codes are employed so that correct decisions can be made.

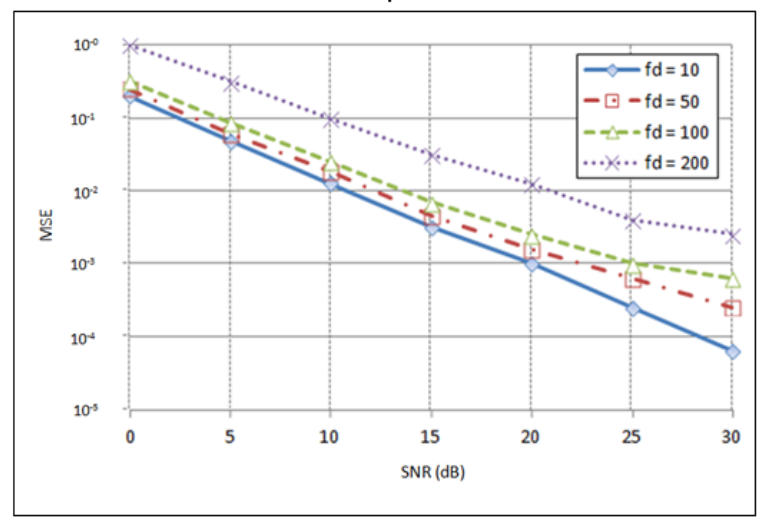

Fig. 2. MSE of channel estimates of the DD EW-RLS estimator with 10\% training data.

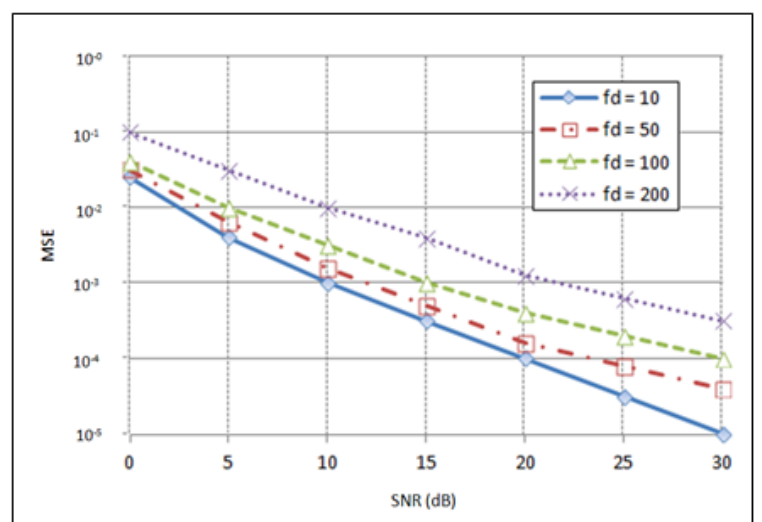

Fig. 3. MSE of channel estimates of the DD EW-RLS estimator with 4\% training data.

It has been shown that the proposed estimator has excellent performance over slowly to moderate time-varying channels and at low SNR. In channels where higher Doppler frequencies are experienced, improved performance can be obtained by increasing the training rates. The estimator is robust and accurate while the complexity of the algorithm is moderate, however, it was noticed that the small values of the forgetting factor of the RLS estimator lead usually to numerical instabilities. Higher training rates lead to better channel tracking and thereby lower error rates. Training symbols need to be sent periodically in order to avoid losing the track, which could occur in case of a deep channel fade. The computational complexity is significantly reduced by recursively updating the channel estimates and by applying the matrix inversion lemma.

\section{B. Outdoor Channel Estimation and Tracking}

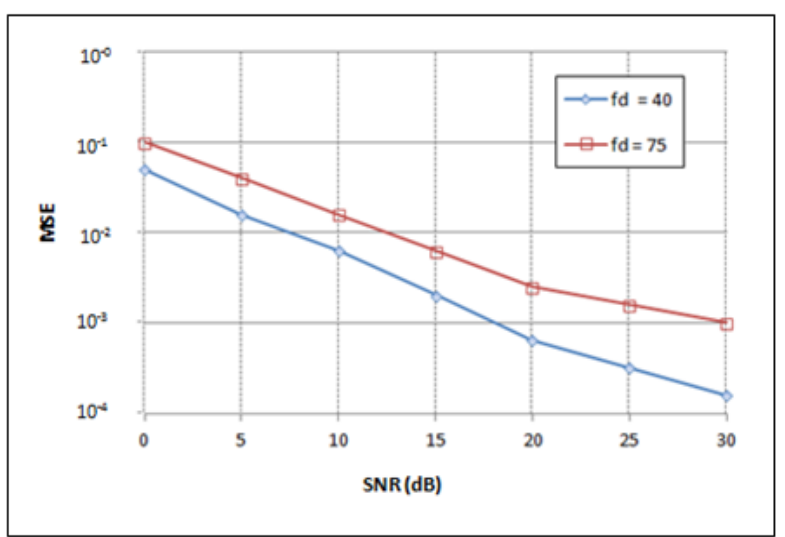

Fig. 4. MSE of channel estimates of the DD EW-RLS estimator with $10 \%$ training data.

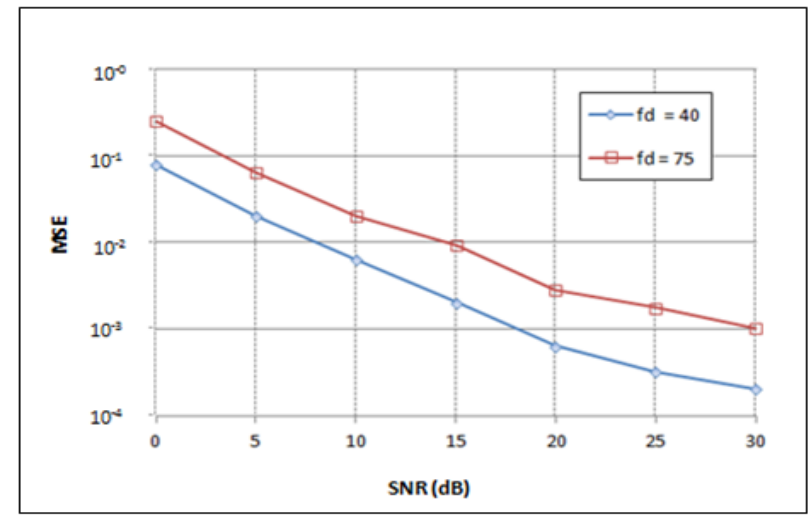

Fig. 5. MSE of channel estimates of the DD EW-RLS estimator with 4\% training data.

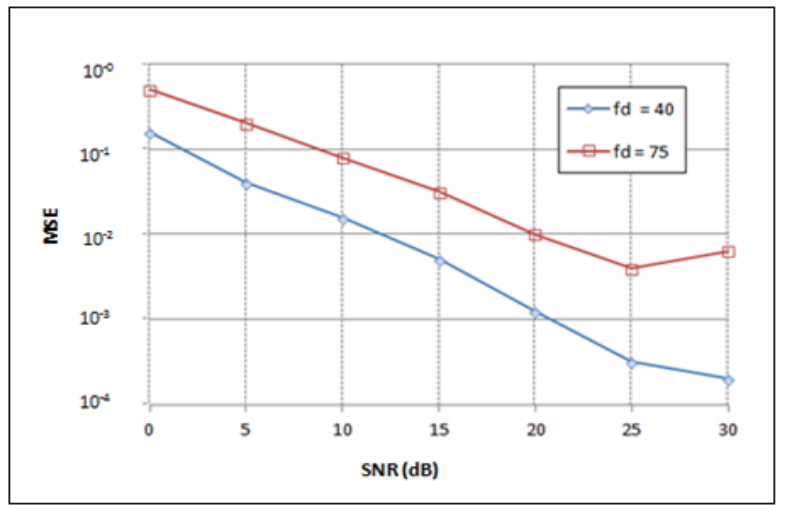

Fig. 6. MSE of channel estimates of the DD EW-RLS estimator with $2 \%$ training data.

Fig. 4 - Fig. 6 show the MSE of the channel tracking versus SNR for training rates of $10 \%, 4 \%$, and $2 \%$, respectively. From the figures, we noticed that as the training rates decreases the estimation errors increases, especially at higher SNR. The increase in MSE pertains to the error propagation resulting from the operation of the channel tracker in the DD-mode. Therefore, it is expected that the tracking capabilities codes are employed so that correct decisions are made.

It has been shown that the proposed estimator has excellent performance over slowly time-varying channels. The proposed estimator adaptively estimates the channel and therefore is robust to channel variations, especially at low 
SNR, while its complexity is moderate. At high SNR, the EW-RLS estimator suffers of the instability problem which leads to some degradation in its performance. Higher training rates lead to better channel tracking and thereby lower error rates. The performance of the EW-RLS algorithm relies extremely on its forgetting factor that needs to be carefully selected for better estimation performance.

\section{CONCLUSION}

The performance of time-domain adaptive channel estimation and tracking scheme based on EW-RLS algorithm has been presented for a MIMO OFDM system. Simulation results are presented in terms of the MSE of channel tracking. It has been shown that the proposed estimator has excellent performance over slowly to moderate time-varying channels and at low SNR. In channels where higher Doppler frequencies are experienced, improved performance can be obtained by increasing the training rates. The estimator is robust and accurate while the complexity of the algorithm is moderate, however, it was noticed that the small values of the forgetting factor of the RLS estimator lead usually to numerical instabilities. Higher training rates lead to better channel tracking and thereby lower error rates. Training symbols need to be sent periodically in order to avoid losing the track, which could occur in case of a deep channel fade. Based on this research, we can conclude that the computational complexity is significantly reduced by recursively updating the channel estimates and by applying the matrix inversion lemma. At high SNR, the EW-RLS estimator suffers of the instability problem which leads to some degradation in its performance. The performance of the EW-RLS algorithm relies extremely on its forgetting factor that needs to be carefully selected for better estimation performance. For future development, OFDM can be replaced with OFDMA to increase the system reliability. Besides that, Space Time Frequency Block Code can be added to make sure the system achieved maximum diversity order.

\section{ACKNOWLEDGMENT}

Authors want to thank all members of Communication Engineering Group for sharing along their knowledge. This research was supported by the Faculty of Electrical Engineering University Teknologi MARA, Shah Alam Selangor, Malaysia.

\section{REFERENCES}

[1] B. Tao and L. Yongling, "Improved space-time-frequency block code for MIMO-OFDM wireless communications," Signal Processing, Communication and Computing, 2012, no. 3, pp. 538-541.

[2] A. Kanchan and S. Dwivedi, "Comparison of BER performance in OFDM using different equalization techniques," International Journal of Engineering and Advanced Technology (IJEAT), no. 6, pp. 139-143, 2012.

[3] M. Rana, "Performance comparison of LMS and RLS channel estimation algorithms for 4G MIMO OFDM systems," in Proc. 14th International Conference on Computer and Information Technology (ICCIT), 2011, pp. 635-639.

[4] Y.-P. Li, T.-S. Lee, and B.-F. Wu, "A variable step-size sign algorithm for channel estimation,” Signal Processing, pp. 10-17, Mar. 2014.

[5] B. B. S. K. Reddy, "Adaptive modulation and coding in COFDM for WiMAX using LMS channel estimator," in Proc. Conference on Advances in Communication and Control Systems, vol. 2013, no. Cac2s, 2013, pp. 23-29.

[6] ETSI, "broadband radio access network (BRAN); HIPERLAN type 2; physical (PHY) layer," TSI TS 101475 v1.1.1 (2000-04), Apr. 2000.

Suzi Seroja Sarnin received her B.Eng. (Hon) from Universiti Teknologi Malaysia (UTM), Malaysia in 1999 and the M.Sc. (microelectronic), from Universiti Kebangsaan Malaysia (UKM), Malaysia in 2006 respectively. She currently pursues her PhD in UiTM. Her research interests include coding algorithm for CDMA and WCDMA, mobile telecommunication, synchronization algorithm in OFDMA for the Wimax technology application and Wimax technology application.

Siti Maisurah Sulong received her B.Eng. (Hon.) in communication from Universiti Teknologi MARA (UiTM) Shah Alam in 2014. She currently pursues her M.Sc (Electrical) in UiTM. Her research interests include wireless telecommunication, OFDMA for the WiMax, equalizers and diversity scheme. 